\title{
Reliable Evaluation of the Lateral Resolution of a Confocal Raman Microscope by Using the Tungsten-dot Array Certified Reference Material
}

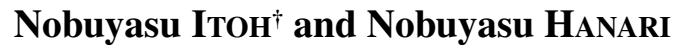 \\ National Metrology Institute of Japan (NMIJ), National Institute of Advanced Industrial Science and Technology \\ (AIST), 1-1-1 Umezono, Tsukuba, Ibaraki 305-8563, Japan
}

\begin{abstract}
Confocal Raman microscopes are widely used in various applications because they provide physical and chemical information at a submicron scale. A high lateral resolution in the confocal Raman microscope is essential for obtaining high-quality images. We used an array of tungsten dots at a $600 \mathrm{~nm}$ pitch on a Si substrate of the certified reference material (NMIJ CRM 5207-a) to reliably evaluate the lateral resolution of a confocal Raman microscope at various pinhole sizes. The precision of the mapping scale in the $x$ - and $y$-pitches was confirmed from Si signal profiles, and the lateral resolution was evaluated by a straight-edge method using scale indicators in the reference material. Because these procedures are applicable to other confocal Raman microscopes with popular specifications (532 nm laser, $100 \times$ objective lens, numerical aperture 0.9, step size $0.1 \mu \mathrm{m}$ ), they are suitable for both a reliable evaluation of the lateral resolution of a confocal Raman microscope and for daily checks on the precision of its mapping scale.
\end{abstract}

Keywords Confocal Raman microscopy, lateral resolution, tungsten-dot array, certified reference material

(Received February 13, 2020; Accepted March 9, 2020; Advance Publication Released Online by J-STAGE March 20, 2020)

\section{Introduction}

Raman spectroscopy is based on the change in frequency of scattered radiation caused by molecular vibrations. It therefore provides information on the physical and chemical properties of a material under noncontact and nondestructive conditions. ${ }^{1-5}$ Confocal Raman microscopy combines Raman spectrometry and laser confocal microscopy to provide information on the physical and chemical properties of a material on a submicron scale. By moving a sample with an $x, y$-stage or by scanning a laser with a galvo mirror, the distributions of materials and states within a sample at a submicron scale can be obtained as a Raman spectral map. Consequently, confocal Raman microscopes have been widely used in such fields as carbon materials, semiconductors, earth sciences, pharmaceuticals, and microplastics. ${ }^{4-6}$

Lateral resolution is one of the most important factors for imaging quality, and a high lateral resolution is necessary to obtain high-quality images. The lateral resolution is dependent not only on the operating principle and specification of the instrument, but also on the conditions for the evaluation (including the skill of the operator), the nature of the sample, and the method of evaluation. Consequently, standard protocols are needed, which are provided in various ISO standards, such as ISO $18516: 2019$ and ISO 18337:2015. ${ }^{7,8}$ On the other hand, there are no standard protocols for evaluating the lateral resolution of a confocal Raman microscope. Although ISO

$\dagger$ To whom correspondence should be addressed.

E-mail: nobuyasu-itoh@aist.go.jp
$18337: 2015^{7}$ for confocal fluorescent microscopes could, in principal, be applied to confocal Raman microscopes, it is possible that use of fluorescent beads might introduce an overestimation of the results due to their much stronger signal intensity compared with Raman signals. It is therefore preferable to evaluate the lateral resolution of a confocal Raman microscope by using Raman signals, although the lateral resolution is also affected by several other factors, even when a Raman signal is used. ${ }^{4,5,10}$ Nanostructured substrates and carbon nanotubes (CNTs) on substrates have been proposed as sample materials for evaluating the lateral resolution of confocal Raman microscopes by using Raman signals. ${ }^{5,11,12}$ These samples should, in principle, provide reasonable estimates of the lateral resolution, since they emit Raman signals and have a sufficiently small size with line shapes or regular structures; however, nanostructured substrates are difficult to obtain, and it is somewhat difficult to find suitable targets in a dispersion of CNTs. Furthermore, reports on studies with these materials contain no description of an examination of the precision of the mapping scale, although this is the most important parameter for achieving a reliable estimate of the lateral resolution; moreover, there is also a possibility of imprecision in the mapping scale due to a change in the step size with time after calibration. ${ }^{5}$

The tungsten-dot array certified reference material NMIJ CRM 5207-a, which is now available from distributors, has been developed for calibrating the magnification of a scanning electron microscope and for measuring its image sharpness. This certified reference material has regular structures of tungsten dots on a Si substrate $(120,200$, and $600 \mathrm{~nm}$ for both $x$ - and $y$-pitches), the pitches of which are provided as certified values. $^{13}$ Because only the $\mathrm{Si}$ substrate is Raman active, 


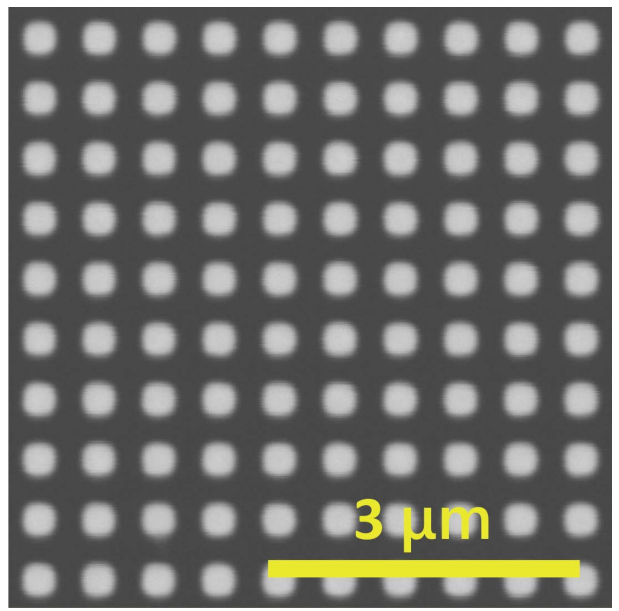

Fig. 1 Scanning electron microscope (SEM) image of a tungsten-dot array with a pitch of $600 \mathrm{~nm}$ in NMIJ CRM 5207-a.

the tungsten dots can be observed as dark spots on a bright background of the $\mathrm{Si}$ signals in the Raman spectral map. Therefore, the precision of mapping scales can be evaluated by comparing the peak-top intervals of the dots with the certified values. Lateral resolutions can be evaluated by means of the straight-edge method using the sharp border between the two different materials; this is one of the popular methods for evaluating lateral resolutions, and is described in ISO 18516:2019. ${ }^{8}$ The reference material NMIJ CRM 5207-a contains scale indicators made of tungsten and lying outside the array area to permit checking of the position and location. The borders of these scale indicators with the $\mathrm{Si}$ substrate are sufficiently clear and sharp to permit their use in the straightedge method with confocal Raman microscopes.

In this study, by using the certified reference material NMIJ CRM 5207-a, we evaluated the precision of the mapping scale of a confocal Raman microscope by using the tungsten-dot array with a pitch of $600 \mathrm{~nm}$, and we then evaluated the lateral resolution of the microscope by a straight-edge method using scale indicators. The suitability of this certified reference material for reliable evaluation of the lateral resolution with confocal Raman microscope is discussed.

\section{Experimental}

\section{Sample and instrument}

The tungsten-dot certified reference material NMIJ CRM 5207-a was obtained from the National Metrology Institute of Japan (NMIJ). Dot-array C (certified value \pm the expanded uncertainty $=597.7 \pm 7.3 \mathrm{~nm}$ for both $x$ - and $y$-pitches, Fig. 1) was used as a sample in this study.

The confocal Raman microscope used was a LabRAM HR Evolution (HORIBA Ltd., Kyoto, Japan) equipped a $532 \mathrm{~nm}$ laser and a $300 \mathrm{gr} / \mathrm{mm}$ grating. The Raman-shift range of spectra obtained by using this system was $100-3200 \mathrm{~cm}^{-1}$. Data were obtained under the following conditions: laser power at $48 \mathrm{~mW}$ with a $100 \times$ objective lens (numerical aperture 0.9 ). A motor-driven $x, y$-stage was used to obtain Raman signal maps and profiles.

\section{Evaluation of the mapping scale}

Raman spectral maps of the tungsten-dot array were obtained

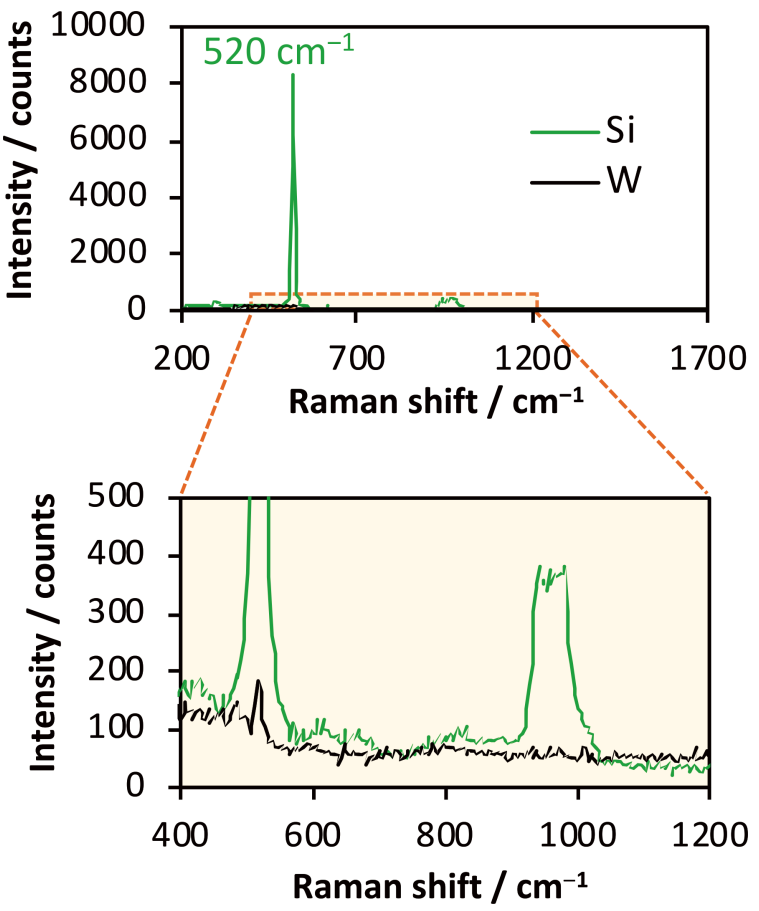

Fig. 2 Typical Raman spectra of $\mathrm{Si}$ and tungsten (W) without binning of the CCD channel. The Si peak at $520 \mathrm{~cm}^{-1}$ was used for subsequent experiments.

at sizes of $3 \times 3 \mu \mathrm{m}$ with a $0.1 \mu \mathrm{m}$ step size at pinhole sizes of 50 and $200 \mu \mathrm{m}$. The exposure times were 0.5 and $0.2 \mathrm{~s}$ for pinhole sizes of 50 and $200 \mu \mathrm{m}$. A single iteration was used, and the CCD binning was set at five channels to increase the signal intensity. Five areas were examined to ensure reproducibility.

Regular pitches of dark spots originated from the tungsten dots were calculated from profiles of the Si peak at $520 \mathrm{~cm}^{-1}$. Peak-top values were obtained by using a Gaussian function, and the intervals between peak tops were evaluated to give the pitches of the tungsten dots.

\section{Evaluation of lateral resolution}

A straight-edge method based on ISO $18516: 2019^{8}$ was used to measure the lateral resolution. Tungsten scale indicators outside the dot array area in the certified reference material were used for the straight-edge method. Raman spectra were obtained at $0.1 \mu \mathrm{m}$ intervals over a $\pm 2 \mu \mathrm{m}$ range from the borders of materials, and were recorded at four different pinhole sizes. The exposure times were $1,0.1,0.05$, and $0.05 \mathrm{~s}$ for pinhole sizes of $25,50,100$, and $200 \mu \mathrm{m}$, respectively, and a single iteration was used. The binning of CCD channels was not applied.

In the straight-edge method, the lateral resolution was evaluated from the distance between the 12 and $88 \%$ intensity points $\left(D_{12-88}\right)$ of the Si peak at $520 \mathrm{~cm}^{-1}$. The $D_{12-88}$ values were estimated by means of a Gaussian function, using the Logistic Function Profile Fitting program from the National Institute of Standards and Technologies (NIST), in accord with ISO 18516:2019. ${ }^{8}$ The lateral resolutions were evaluated for five different scale indicators at each pinhole size. 
(a)

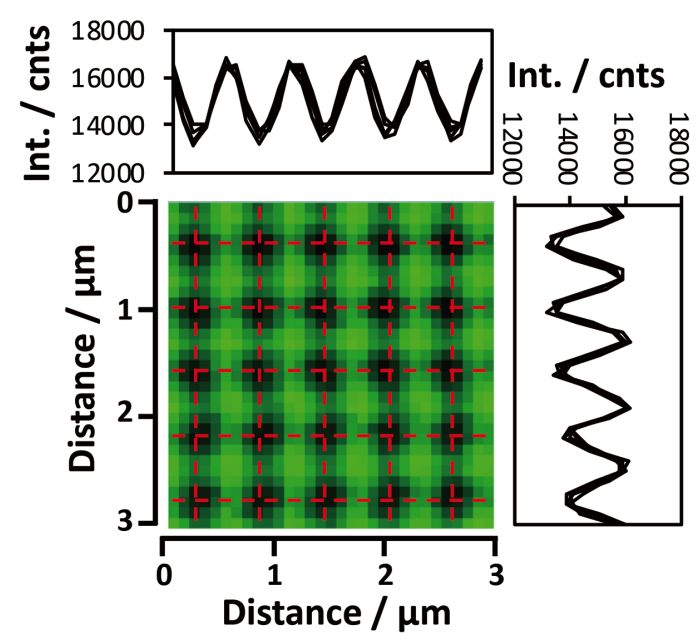

(b)

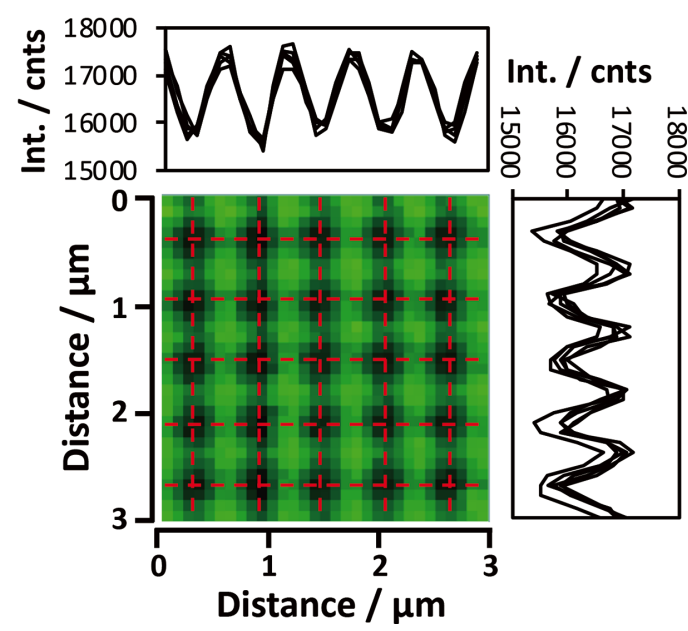

Fig. 3 Raman spectral maps of the $\mathrm{Si}$ signal at $520 \mathrm{~cm}^{-1}$ and their profiles obtained pinhole sizes of $50 \mu \mathrm{m}$ (a) and $200 \mu \mathrm{m}$ (b). The green-colored areas correspond to $\mathrm{Si}$, and the signal profiles were obtained from the area defined by the red dashed lines on the maps. For interpreting the references to color in this figure caption, the reader is referred to the web version of this article.

\section{Results and Discussion}

Raman spectral map of the tungsten-dot certified reference material

The tungsten-dot reference material NMIJ CRM 5207-a consists of an array of tungsten dots on a Si substrate at three different pitches $(120,200$, and $600 \mathrm{~nm})$. Of these three different pitches, we used the $600 \mathrm{~nm}$ pitch because $0.1 \mu \mathrm{m}$ is the most popular minimum step size for both motor-driven $x, y$ stages and galvo mirrors and the 120 and $200 \mathrm{~nm}$ pitches are too small to permit an evaluation at a $0.1 \mu \mathrm{m}$ step size. We used both 50 and $200 \mu \mathrm{m}$ pinhole sizes, because the lateral resolution of a confocal Raman microscope is dependent on the pinhole sizes at a constant wavelength and numerical aperture of the objective lens, and a smaller pinhole sizes provides a better lateral resolution. ${ }^{4,5,11,12}$

Figure 2 shows typical Raman spectra of $\mathrm{Si}$ and tungsten obtained from outside the dot-array area without CCD binning. $\mathrm{Si}$ produces a strong signal at $520 \mathrm{~cm}^{-1}$ corresponding to its first-order phonon band, whereas tungsten does not produce any signal at $520 \mathrm{~cm}^{-1}$. Because no large peaks were observed near (a)

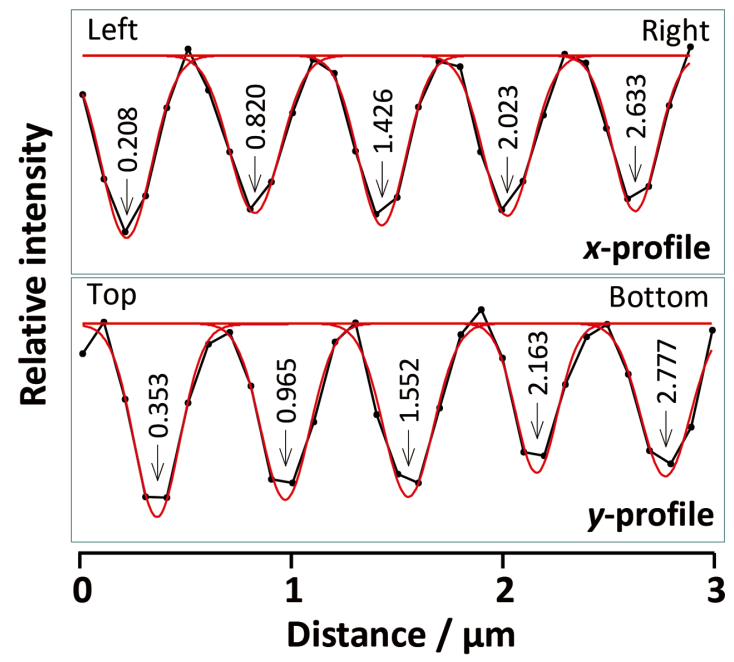

(b)

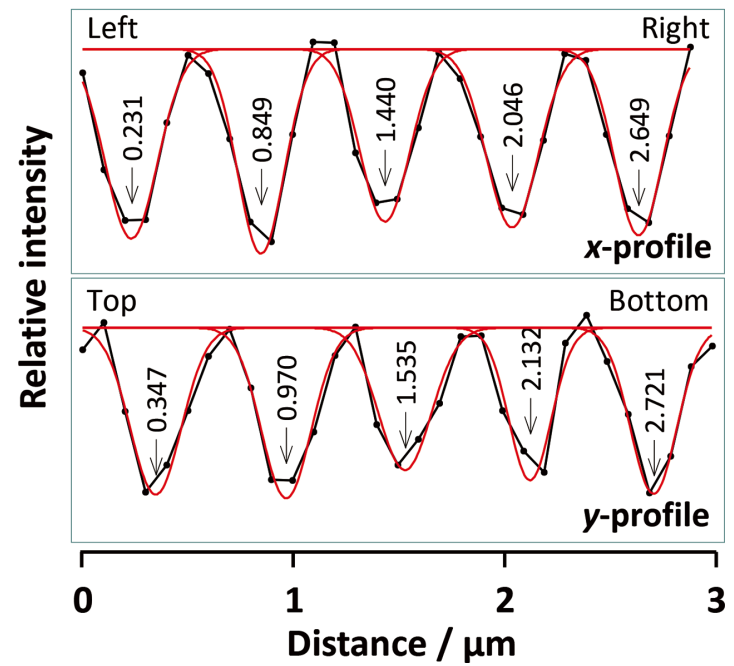

Fig. 4 Si signal profiles and their fitting results at the third $x$-profile and third $y$-profile obtained at pinhole sizes of $50 \mu \mathrm{m}$ (a) and $200 \mu \mathrm{m}$ (b). Black, data; red, fitted curve. For interpreting the references to color in this figure caption, the reader is referred to the web version of this article.

$520 \mathrm{~cm}^{-1}$ for either Si or tungsten, we applied the binning of five CCD channels to increase the signal intensity and to reduce the time required to obtain Raman spectral maps.

Figure 3 shows Raman spectral maps of the Si signal at $520 \mathrm{~cm}^{-1}$, with their $x$ - and $y$-profiles obtained at pinhole sizes of 50 and $200 \mu \mathrm{m}$. Regular dark spots in both the $x$ - and $y$ pitches $(5 \times 5$ dark spots in each map) were observed; however, no clear differences were observed in the maps obtained at pinhole sizes of 50 and $200 \mu \mathrm{m}$, although the lateral resolution must be affected by the difference in the pinhole size. ${ }^{4,5,12}$ All profiles obtained were also comparable for both the $x$ - and the $y$-pitches at pinhole sizes of 50 and $200 \mu \mathrm{m}$. The observation of six points between the two minimum points through a maximum for all profiles was reasonable, because the $600 \mathrm{~nm}$ dot pitches were evaluated with a $0.1 \mu \mathrm{m}$ step size (also see below). Although no obvious differences were observed in the two sets of maps and profiles, the intensity of the Raman signal at a 
Table 1 Measured $x$ - and $y$-pitches of dark spots for pinhole sizes of 50 and $200 \mu \mathrm{m}$

\begin{tabular}{cccc}
\hline Pinhole size $/ \mu \mathrm{m}$ & Order & $x$-Pitch $/ \mu \mathrm{m}$ & $y$-Pitch $/ \mu \mathrm{m}$ \\
\hline \multirow{2}{*}{50} & 1st & $0.608(0.010)$ & $0.600(0.005)$ \\
& 2nd & $0.601(0.011)$ & $0.604(0.008)$ \\
& 3rd & $0.606(0.007)$ & $0.606(0.013)$ \\
& 4th & $0.606(0.016)$ & $0.606(0.014)$ \\
\multirow{2}{*}{200} & 5th & $0.598(0.007)$ & $0.602(0.008)$ \\
& 1st & $0.603(0.019)$ & $0.589(0.011)$ \\
& 2nd & $0.601(0.033)$ & $0.593(0.024)$ \\
& 3rd & $0.605(0.011)$ & $0.594(0.024)$ \\
& 4th & $0.595(0.005)$ & $0.584(0.007)$ \\
& 5th & $0.600(0.015)$ & $0.589(0.039)$ \\
\hline
\end{tabular}

a. The values in parentheses are standard deviations for four peak intervals obtained for five peaks.

pinhole size of $200 \mu \mathrm{m}(0.2 \mathrm{~s})$ was higher than that at $50 \mu \mathrm{m}$ $(0.5 \mathrm{~s})$.

\section{Evaluation of the precision of the mapping scale}

To evaluate dot pitches of dark spots in maps and profiles, each peak-top (darkest spot of tungsten) was initially evaluated by using a Gaussian function. Figure 4 shows Si signal profiles of the third lines and third columns in Figs. 3(a) and 3(b) with their fitting results as examples. As shown in these figures, the fitting curves matched the signal intensity profiles well; this was attributed to the use of a sufficient number of points ( 6 points/ peak), and the intervals between the peak tops (values shown in Figs. 4(a) and 4(b)) commonly showed a $0.6 \mu \mathrm{m}$ pitch in all profiles. These profiles and fitting results showed no differences in the pitches measured at pinhole sizes of 50 and $200 \mu \mathrm{m}$.

Table 1 summarizes the evaluated dot pitches for different profiles obtained from the Raman spectral maps (Fig. 3). The measured dot pitches ranged from 0.598 to $0.608 \mu \mathrm{m}$ for a $50 \mu \mathrm{m}$ pinhole and from 0.584 to $0.605 \mu \mathrm{m}$ for a $200 \mu \mathrm{m}$ pinhole. Their repeatability was within $0.02 \mu \mathrm{m}$ (standard deviation of four peak intervals) for the $50 \mu \mathrm{m}$ pinhole and within $0.04 \mu \mathrm{m}$ for the $200 \mu \mathrm{m}$ pinhole size. No clear differences were observed in dot pitches and their repeatability between the $x$ - and the $y$-pitches, although data were obtained line by line. Because the certified values of both the $x$ - and $y$ pitches are $597.7 \pm 7.3 \mathrm{~nm}$, the mapping scale must be reasonable and precise in both the $x$ - and $y$-pitches over the mapping areas. Because comparable and repeatable values in the $x$ - and $y$-pitches were also obtained for four other areas with both the 50 and $200 \mu \mathrm{m}$ pinhole sizes, the mapping scale of our system must be sufficiently precise to permit any reliable evaluation of the lateral resolution.

Evaluation of lateral resolution with the straight-edge method by using scale indicators

ISO 18516:2019 ${ }^{8}$ describes three different method for evaluating the lateral resolution: the straight-edge method, the narrow-line method, and the grating method. Of these three methods, we chose to use the straight-edge method because it is the only one that is applicable to scale indicators having suitable straight-borders with a Si substrate outside the array area in the certified reference material. In using the straight-edge method, the lateral resolution is evaluated as a sharpness parameter, $D_{12-88}$, that characterizes the (sigmoidal) edge-spread function. $D_{12-88}$ is obtained from the distance between the relative signal intensities of 12 and $88 \%$. (a)

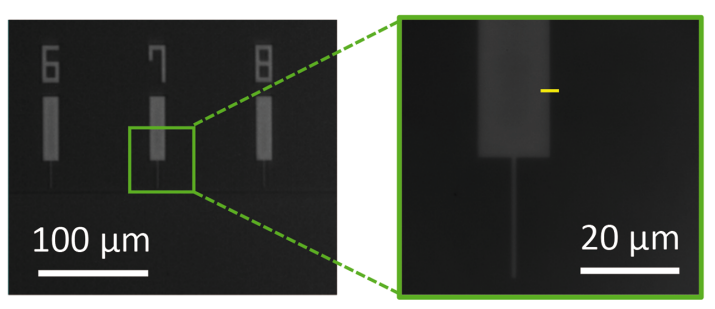

(b)

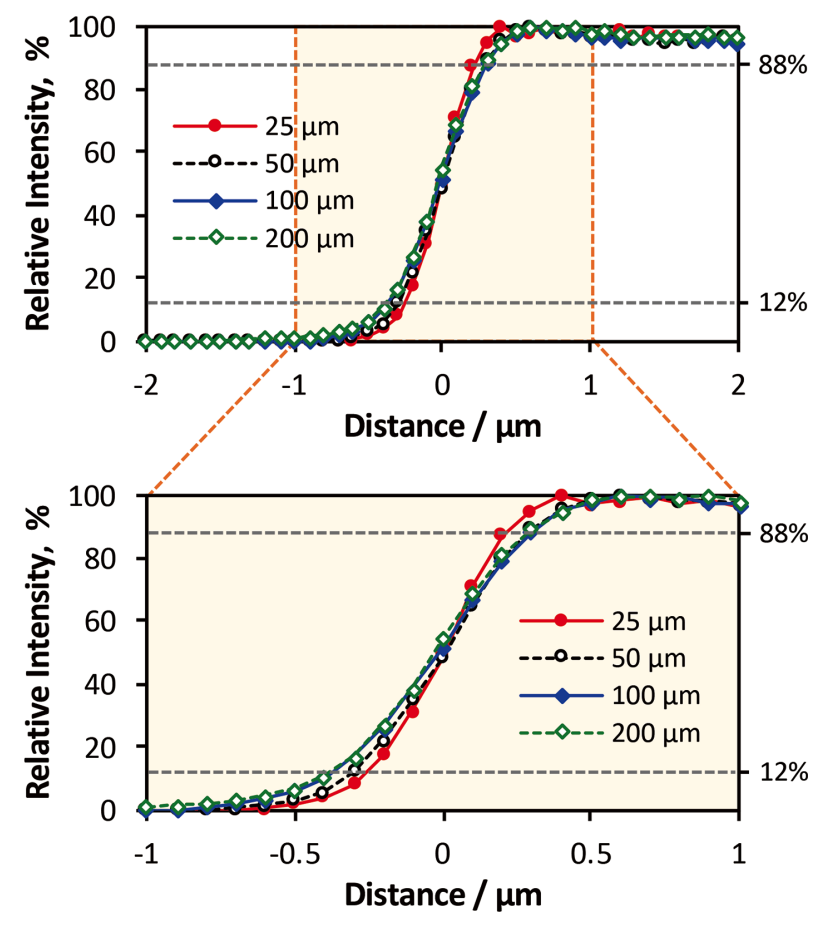

Fig. 5 Typical optical images of a scale indicator obtained with 10x (left) and $100 \times$ (right) objective lenses (a), and typical Si signal profiles of this line obtained at pinhole sizes of $25,50,100$, and $200 \mu \mathrm{m}$ (b). The short yellow line in Fig. 5(a) (right) was an example used for the straight-edge method and corresponds to the profiles in Fig. 5(b).

Figure 5(a) shows the optical image of a scale indicator and Fig. 5(b) shows the intensity profiles of the Si peak at $520 \mathrm{~cm}^{-1}$ for pinhole sizes of 25, 50, 100, and $200 \mu \mathrm{m}$. In Fig. 5a, the scale indicators of the certified reference material (white areas, tungsten) can be clearly recognized from optical images obtained with the $10 \times$ and $100 \times$ objective lenses, and their borders are sufficiently sharp. The signal profiles unaffected by noise (Fig. 5(b)) were obtained with a high-power laser at a short exposure time (from $0.05 \mathrm{~s}$ for pinhole sizes of 100 or $200 \mu \mathrm{m}$ to $1 \mathrm{~s}$ for a pinhole size of $25 \mu \mathrm{m}$ ) because $\mathrm{Si}$ and tungsten are less susceptible to damage with a high-power laser. The $\mathrm{Si}$ signal intensity started to increase from the -1.0 to $+0.6 \mu \mathrm{m}$ point and reached $100 \%$ at a plateau. The signal intensity for the $25 \mu \mathrm{m}$ pinhole size increased most dramatically; this was followed by that at the $50 \mu \mathrm{m}$ pinhole size, whereas profiles for pinhole sizes of 100 and $200 \mu \mathrm{m}$ were similar.

Table 2 summarizes the $D_{12-88}$ values obtained for each pinhole size, together with the calculated pinhole areas. The obtained $D_{12-88}$ values were $0.426,0.536,0.631$, and $0.642 \mu \mathrm{m}$ for pinhole sizes of $25,50,100$, and $200 \mu \mathrm{m}$, respectively. Because more 
Table $2 \quad D_{12-88}$ values obtained with various pinhole sizes

\begin{tabular}{ccc}
\hline Pinhole size $/ \mu \mathrm{m}$ & Pinhole area/ $\mu \mathrm{m}^{2}$ & $D_{12-88} \mathrm{a} / \mu \mathrm{m}$ \\
\hline 25 & 491 & $0.426(0.010)$ \\
50 & 1963 & $0.536(0.036)$ \\
100 & 7854 & $0.631(0.048)$ \\
200 & 31416 & $0.642(0.043)$ \\
\hline
\end{tabular}

a. The values in parentheses are standard deviations for quintuple analyses.

than four plots were used in the $D_{12-88}$ range (from 12 to $88 \%$ ), even for a $25 \mu \mathrm{m}$-pinhole size $(0.426 \mu \mathrm{m}$ with $0.1 \mu \mathrm{m} / \mathrm{plots})$, this condition meets the criteria described in ISO 18516:2019 (plots $\geq 4$ ) with a Gaussian function. ${ }^{8}$ The $D_{12-88}$ values increased with increasing pinhole size, as expected from the signal profiles between 25 and $100 \mu \mathrm{m}$. It is reasonable that smaller pinhole sizes provide information on a smaller volume (area in plane). ${ }^{4,5,9-12}$ On the other hand, there were no clear differences in the $D_{12-88}$ values for pinhole sizes of 100 and $200 \mu \mathrm{m}$, although the area of a pinhole size of $100 \mu \mathrm{m}$ is fourtimes larger than that at $200 \mu \mathrm{m}$. This result suggests that a pinhole size at $200 \mu \mathrm{m}$ is not a limiting factor for the lateral resolution under the examined conditions. In other words, any pinhole size combined with a $532 \mathrm{~nm}$ laser and a $100 \times$ objective lens (N.A. 0.9) can be used for the $600 \mathrm{~nm}$ pitches of the certified reference material. The $D_{12-88}$ value at a pinhole size of $50 \mu \mathrm{m}(0.536 \mu \mathrm{m})$ is comparable (1.2-times greater) to that for a pinhole size of $200 \mu \mathrm{m}(0.642 \mu \mathrm{m})$. Therefore, the differences in the Raman spectral maps in Figs. 3(a) and 3(b) are unclear.

\section{Conclusions}

The 600-nm-pitch tungsten-dot array in the certified reference material a NMIJ CRM 5207-a was used for a reliable evaluation of the lateral resolutions of a confocal Raman microscope. The precision of the mapping scale was evaluated from the dot pitches of dark spots corresponding to tungsten dots, and the lateral resolution was evaluated by using scale indicators in conjunction with the Raman signal of the $\mathrm{Si}$ substrate. This certified reference material is easily obtained from distributors, and also provides a target sample that does not undergo burning by the laser of the microscope. Consequently, this reference material is suitable not only for reliable evaluations of the lateral resolution of confocal Raman microscopes, but also for daily checks of their mapping scales.

\section{References}

1. R. L. McCreery, "Raman Spectroscopy for Chemical Analysis", 2000, Wiley Interscience, New York.

2. I. R. Lewis and H. Edwards, "Handbook of Raman Spectroscopy: From the Research Laboratory to the Process Line (Practical Spectroscopy)", 2001, CRC Press, New York.

3. J. R. Ferraro, K. Nakamoto, and C. W. Brown, "Introductory Raman Spectroscopy", 2nd ed., 2003, Academic Press, Amsterdam.

4. A. Zoubir, "Raman Imaging: Techniques and Applications", 2012, Springer, Cham.

5. J. Toporski, T. Dieing, and O. Hollricher, "Confocal Raman Microscopy", 2nd ed., 2019, Springer, Cham.

6. C. F. Araujo, M. M. Nolasco, A. M. P. Ribeiro, and P. J. A. Ribeiro-Claro, Water Res., 2018, 142, 426.

7. ISO18337:2015, "Surface Chemical Analysis-Surface Characterization-Measurement of the Lateral Resolution of a Confocal Fluorescence Microscope".

8. ISO18516:2019, "Surface Chemical Analysis-Determination of Lateral Resolution and Sharpness in Beam Based Methods with a Range from Nanometres to Micrometres".

9. N. J. Everall, Analyst, 2010, 135, 2512.

10. N. J. Everall, Appl. Spectrosc., 2009, 63, 245A.

11. J. Park, J. Kim, and H. Kwon, Bull. Korean Chem. Soc., 2020, 41, 34.

12. Y. Kim, E. J. Lee, S. Roy, A. S. Sharbirin, L.-G. Ranz, T. Dieing, and J. Kim, Curr. Appl. Phys., 2020, 20, 71.

13. National Metrology Institute of Japan (NMIJ), "Reference Material Certificate-NMIJ CRM 5207-a (Tungsten Dotarray)", 2018, Tsukuba. 